\title{
A Quantitative Analysis for Inter-individual Knowledge Transferring Obstacle
}

\author{
Dehai Liu ${ }^{1,2}$ Weiguo Wang ${ }^{1}$ \\ ${ }^{1}$ School of mathematics and Quantitative Economics, Dongbei University of Finance \& Economics, Dalian 116025, \\ P. R. China \\ ${ }^{2}$ School of Economics and Management, Xi’an Technological Univresity, Xi’an 710032, P.R. China
}

\begin{abstract}
This paper puts forward a quantitative analysis method measuring inter-individual knowledge transferring obstacle based on evolutionary game theory. According to economic exchange theory, the key influencing factors on the inter-individual knowledge transfer includes the expected incomes and transfer obstacles of different knowledge. This paper brings forward the knowledge transfer model among the different social departments and a quantitative method to indirectly measure the corresponding knowledge transfer obstacles among various departments. Then it gives a case that shows how the knowledge transferring obstacles among Chinese various ruralurban departments are measureed. At last, the paper points out some shortcomings of this analytical method.
\end{abstract}

Keywords: Knowledge transferring obstacle, Evolutionary game, General replicator dynamic, Quantitative analysis

\section{Introduction}

Along with entering the knowledge economy epoch, international scholars extensively research the knowledge diffusion and transferring. Knowledge consists in the different organizational layers, so the key of knowledge management is finding out the knowledge characteristics and transferring approaches among the different organizational layers. Knowledge transferring is divided into three layers including individual, group and organization (inter-organization and inner-organization) in the existing researching papers. Earl Michael distinguishes the data, information and knowledge on the viewpoint of content, format, primary task, artificial factors, organizational target and performance, and divides knowledge into two states that's "what you know" and “what you don't know”, and the cognizing degrees of knowledge include explicit knowledge, tacit knowledge, planned ignorance knowledge and innocent ignorance knowledge [1]. Ikujiro Nonaka puts forward the SECI model and the concepts of explicit knowledge and tacit knowledge, where the four exchange modes between explicit knowledge and tacit knowledge are Socialization, Externalization, Combination and Internalization [2]. The researches about informal inter-individual knowledge transferring pay more attention to the three key affects including individual characteristic, knowledge characteristic and inter-individual relationship characteristic, that those key factors have effect on the incentive, manner and result of knowledge transferring [3]-[4]. Danie puts the OADI-SMM (Observe-Assess-Design-Implement with Shared Mental Models) model to expound the interaction between individual learning and organizational learning [5]. Gunnar Hedlund researches the inter-organizational knowledge and divides it into the tacit and articulated two types [6]. D. Croasdell puts forward the Organizational Memories model that can store dynamic knowledge within organization, and organizational member could obtain some special knowledge without restriction [7]. To make a summary about above papers, the existing researches discuss knowledge characteristic, transferring mechanism on the different layers and different types from the theoretical analysis angle. To the best of our knowledge, however, there doesn't make the quantitative analysis about knowledge transferring.

Besides many theoretical literatures on knowledge transferring obstacles, domestic scholars make the empirical qualitative analysis that has two methods: one is qualitative analysis the inscapes of knowledge transferring obstacles, and the other is combination some quantitative analysis method as a semi-structured model. Xu Jin-fa etc. put the scene model about enterprise knowledge transferring on the dimensionality of culture, strategy, organizational structure and course, environment, technology and operation [8]. Wang Zhao-xiang builds the Hierarchical Model about the knowledge transferring course that includes physical layer, data layer, language layer, knowledge layer, capability layer and application layer [9]. Peng Can analyzes the three types knowledge transferring obstacles in the region 
innovating system there are knowledge obstacle, system obstacle and organizational obstacle [10]. Wang Ying-luo etc. puts that knowledge transferring course has two manners including the language modulate and the coupling learning, and tacit knowledge can be divided into really tacit and feignedly tacit [11]. Wang Tong-an etc. takes Structure Equation Model to analyze the quantitative relationship among credit, interpersonal relationship, incentive, decision-maker's attitude, knowledge management system and knowledge absorbency through investigating many enterprises' data [12]. The above existing domestic literatures show two analytical methods about knowledge transferring obstacles: one is constructing the qualitative analytical model reflecting the various influencing factors; the other is constructing the semi-structure model to make the empirical quantitative analysis based on the questionnaire survey. However, for a large-scale knowledge transferring phenomenon in social system, it's not only difficult to collect data in this empirical quantitative analysis, but also difficult to build the appropriate structure model for the complicated social economy system that need combine artificial estimation.

Arising in the 1990's, evolutionary game theory analyzes the group learning behavior based on the bounded rationality hypothesis that provides a new theoretical method to analyze the evolving origin, mechanism, course and stability for social economy complicated system. There have many social learning model describing the individual learning and social learning. The article puts forward a quantitative analysis method measuring inter-individual knowledge transferring obstacle based on evolutionary game theory. In the first part, it introduces the learning course in evolutionary game; secondly, it puts a indirectly quantitative measuring method about knowledge transferring obstacle based on general replicator dynamic model; thirdly, as a applied case it measures the knowledge transferring obstacles among Chinese different rural-urban economy departments. At last, the paper points out some shortcomings of this analytical method.

\section{Learning Course in Evolutionary Game}

\subsection{Basic Hypothesis Evolutionary Game}

The traditional game theory thinks that the equilibrium is come from the player's rational analysis and introspection under the game rule, player's rationality and payoff function are the common knowledge. This strong rational hypothesis is criticized by the more and more economists and game theorists and put forward another method reached to the equilibrium that is the long-term learning result when the bounded rational players seeks the equilibrium.

Evolutionary game theory is come of biology colony evolutionary phenomenon in the 1980's. In the first instance, it analyzes the colony competition course in biological system that the different behavior characteristics compete with each other and screen out the succeeding characteristic possessing the higher reproduction rate, that's described as fitness function, by the inheritance and variance mechanism.

The theory applies into social economy system evolutionary phenomena since the 1990's. For the repeated game among large group, some players are chosen to make the factor game as a representative of the social group taking the same strategy in the random sampling course. The structure of factor game has been regulated in advance. Because the player couldn't know about the total game structure, his (her) behavior manner embodies the bounded rational characteristics such as inertia, myopia, trial and errors experiments, and imitates the other higher payoff strategy when choosing the next phase action. At last, the bounded rational groups will also reach the equilibrium state by the long-term learning course that the time continuous adjustment remedies the lack of rational ability.

\subsection{Various Types of Learning Course}

The hallmark of an evolutionary model is inclusion two processes: one is the selection process, and the other is the mutation process [13]. Therein, the basic hypothesis of selection dynamic is satisfied to payoff monotonicity: for any two strategies yielding unequal payoffs, the higher payoff one has the higher growth rate. Based on the different rational abilities, selection dynamic includes various types: payoff-positive dynamic, convex-monotone dynamic, weakly payoffpositive dynamic, etc. Nachbar (1990) analyzed payoff-positive dynamic, where all pure strategies earning above average have positive growth rates and those earning below average have negative growth rates [14]. Weibull (1995) analyzed weakly payoffpositive dynamic, where at least one pure strategy that earns above average has a positive growth rate when such a strategy exists [13]. A familiar selection dynamic is Replicator Dynamics that one player insists on long-term taking the same strategy $i$ because of the transforming cost for the new strategy, and the increasing rate of population distributional proportion 
taking this strategy $\dot{\theta}_{i}(t)$ is a strict increasing function of difference between the utility $\pi_{t}\left(\theta_{i}\right)$ of strategy $i$ and the total average utility $\bar{\pi}_{t}$ :

$$
\dot{\theta}_{i}(t)=\theta_{i}(t) \cdot\left[\pi_{t}\left(\theta_{i}\right)-\bar{\pi}_{t}\right]
$$

Some familiar adaptability learning mechanisms are following:

(1) Reinforcement learning. The probability of taken one strategy is increasing along with its foregone payoff increasing. So the player is inclined to take the high payoff strategy in the past, and avoids the low payoff strategy.

(2) Best-response dynamic. Players expect the others have the different rational degree and maximize his expected payoff. A simple model is players choose the best-response based on the others former action experiential frequency distribution, that's fictitious play game model.

(3) Nature selection or social simulation. This mechanism is the boundedly rational population learning behavior in evolutionary game theory. The player more easily succeeds to replicate the own strategy when this strategy has the higher payoff based on the gene inheritance mechanism. So this strategy has the higher adaptability in the survival competition.

\section{An Indirect Quantitative Measuring Method about Knowledge Transferring Obstacle}

\subsection{A Socialization Learning Model: General Replicator Dynamic}

Nature selection mechanism based on gene inheritance and variation is fitter for explanation to the biological colony evolutionary phenomenon, but the rationality level is very low in this mechanism. So in order that colony behaviors are convergence to the stable equilibrium state, it need quite long term, that's in the geologic era criterion. Evidently, this mechanism isn't fit for the social economy evolutionary phenomenon because of human being's higher rationality level.

For the social economic evolutionary system, there are several alternative mechanisms that can produce some selection dynamic course with the appropriate degrees of inertia. For example, Agents with different types of economic behavior can enter and exit; market outcomes (or outcomes from other economic institutions) can redistribute resources among different types of agents; and individual can change behaviors as they accumulate experiences.
Sethi (1998) considers the different strategies have some specifically learning obstacles, when selection dynamic is applied to the social economy problems [15]. Besides searching cost and deposition cost when players transform the former behavior to the new behavior with the higher payoff, the new behavior itself has some learning obstacle too. In commonly, a behavior with the higher income is easy to be observed by the others in the group, but this behavior often has the larger entering obstacle at the same time, so the increasing rate of the number taking this behavior isn't the highest.

In social economy evolutionary problems, considering the different strategies have the corresponding learning obstacles $\lambda_{i}, \lambda_{j}$, Sethi (1998) thinks that the monotonicity hypothesis in Replicator Dynamic (1) is excessively strict, and builds General Replicator Dynamic Model based on Bjornerstedt and Weibull model, which allows for the fact that some strategies may be harder to learning than others.

Supposed that the population proportion taking the strategy $i$ in time $t$ is $\theta_{i}(t)$, and corresponding payoff is $\pi_{t}\left(\theta_{i}\right)$. Let time be divided into a sequence of discrete intervals of length $\tau$, and suppose that in each interval a proportion $\tau$ of the population consider revising their strategy. They do so by picking at random a member of the population at large and attempting to learn their strategy. The probability that an individual currently using strategy $j$ will be picking is then simply given by the population share $\theta_{j}$. We shall assume that if someone picking strategy $j$ observes its payoff to be at least as high as their own current payoff, they switch to strategy $j$ with probability proportional to $\left(\pi_{j}-\pi_{i}\right)$. By suitable normalization of payoffs, this probability can be set equal to $\left(\pi_{j}-\pi_{i}\right)$. Putting together, the proportion of those adopting strategy $i$ who switch to strategy $j$ during a period of length $\tau$ is given by $p_{i}^{j}$, where

$$
p_{i}^{j}=\left\{\begin{array}{cc}
\tau \lambda_{j}\left(\pi_{j}-\pi_{i}\right) \theta_{j} & \text { if } \pi_{j}>\pi_{i} \\
0 & \text { otherwise }
\end{array}\right.
$$

The set $B_{i}(\theta)=\left\{j \in I \mid \pi_{j}(\theta)>\pi_{i}(\theta)\right\}$ consists of the strategies which yield strictly higher payoffs than strategy $i$ when the population composition is $\theta_{i}(t)$. Now consider the population shares at time $(t+\tau)$ : 


$$
\begin{aligned}
\theta_{i}(t+\tau)=\theta_{i}(t) & +\sum_{j \notin B_{i}(\theta)} \tau \lambda_{i}\left(\pi_{i}-\pi_{j}\right) \theta_{i}(t) \theta_{j}(t) \\
& -\sum_{j \in B_{i}(\theta)} \tau \lambda_{j}\left(\pi_{j}-\pi_{i}\right) \theta_{j}(t) \theta_{i}(t)
\end{aligned}
$$

The above equation can be rearranged to yield:

$$
\begin{aligned}
\frac{\theta_{i}(t+\tau)-\theta_{i}(t)}{\tau}= & \theta_{i}(t)\left\{\sum_{j \notin B_{i}(\theta)} \tau \lambda_{i}\left(\pi_{i}-\pi_{j}\right) \theta_{j}(t)\right. \\
& \left.-\sum_{j \in B_{i}(\theta)} \tau \lambda_{j}\left(\pi_{j}-\pi_{i}\right) \theta_{j}(t)\right\}
\end{aligned}
$$

Taking the limit as $\tau \rightarrow 0$ yields the General Replicator Dynamic:

$$
\begin{aligned}
\dot{\theta}_{i}(t)=\theta_{i}(t) & \cdot\left[\sum_{j \notin B_{i}(s)} \lambda_{i} \cdot\left(\pi_{i}-\pi_{j}\right) \cdot \theta_{j}\right. \\
& \left.-\sum_{j \in B_{i}(s)} \lambda_{j} \cdot\left(\pi_{j}-\pi_{i}\right) \cdot \theta_{j}\right]
\end{aligned}
$$

The first component on the right side of this equation represents those individuals who are switching to strategy $i$ from other, less highly rewarded, strategies. The second component represents those switching away from strategy $i$ to more highly rewarded strategies.

\subsection{An Indirect Quantitative Measuring Method about Knowledge Transferring Obstacle Using the Socialization Learning Model}

Haghirian(2003) thinks knowledge transferring course as a simple communication model. This model includes message, sender and receiver, where sender sends the knowledge codified suitable information form to receiver by the transferring channel, and receiver incepts the information and decodes it, then receiver comprehends the knowledge under own environment [16]. Based on above communicational model, the inter-individual knowledge transferring obstacle includes the knowledge characteristic obstacle and cognizing obstacle between the sender and receiver.

(1) Knowledge characteristic obstacle

Special situation is an important factor influencing knowledge transferring. The formal knowledge intercommunion system based on the IT technology is unsuccessful to the tacit knowledge, because the tacit knowledge that needs more social factors is more difficult to diffusion than the codified knowledge [17]. Uzzi and Lancaster researches the knowledge transferring between officials providing a loan and enterprisers, and finds that the different types of knowledge are corresponding to different learning mechanisms, where the private knowledge transferring further relates to exploration learning mechanism [18].

(2) Cognizing obstacle between the sender and receiver

The motivation of inter-individual knowledge transferring includes economy motivation and social motivation. Hippel(1987) finds that the individual expected economy income has effect on the transferring decision-making [19].

From the angle of economy exchange theory, inter-individual knowledge transferring is a learning course that player imitates the higher payoff strategy, and knowledge transferring obstacle is namely the learning obstacle in the imitating course. The socialization learning model in evolutionary game theory (5) provides the analytical foundation of interindividual knowledge transferring on the viewpoint of economy exchange. Different of the Sethi's explanation, the knowledge transferring obstacle is not only including the probability to be observed, but also other influencing factors, for example, the interindividual cognitive difference. So the transferring obstacle $\lambda_{i}, \lambda_{j}$ in formula $(5)$ isn't $(0,1)$ that the more large this value, the easier knowledge transferring. This model includes some important factors influencing the knowledge transferring direction and speed these are the different expected knowledge incomes $\pi_{i}, \pi_{j}$, corresponding transferring obstacles $\lambda_{i}, \lambda_{j}$ and population proportional distribution among the different types knowledge.

Supposed that some social economy system could be divided into i departments, $i=2, \cdots, n$, knowledge among various departments are transferring through the inter-individual learning course. So we can indirectly measure the knowledge transferring obstacles among various social departments based on the socialization learning model (5), after computed the population proportion and expected economy income in various social departments. Taking some time interval, usually one year, to analysis, we need transform differential equation (5) into following difference equations, that's knowledge transferring dynamic model about social various departments:

$$
\begin{array}{r}
\Delta \theta_{i}(t)=\theta_{i}(t) \cdot\left[\sum_{j \notin B_{i}(s)} \lambda_{i} \cdot\left(\pi_{i}-\pi_{j}\right) \cdot \theta_{j}\right. \\
\left.-\sum_{j \in B_{i}(s)} \lambda_{j} \cdot\left(\pi_{j}-\pi_{i}\right) \cdot \theta_{j}\right]
\end{array}
$$




\section{A Case: Measurement of Knowledge Transferring Obstacles among Chinese Rural- urban Employment Departments}

\subsection{Motivation and Behavior Characteristic of Rural Labor Transfer}

In developing countries, the traditional structure of labor market includes rural department, urban informal department and urban formal department [20]. Hu Angang puts forward the concept of informal employment and formal employment, where formal employment refers to urban staff and workers, and informal employment is mostly constituted by three parts: small and medium private enterprise, family workshop and self-employed individuals [21]. The famous Todaro model in developing economics thinks rural labor transfer is reflected of expected rural-urban income difference. Rural labors gradually transfer from rural department to urban department for seeking the higher income along with knowledge transferring including the ability obtaining urban employment and life style.

But, Zhao Yao-hui finds that non-agricultural industries in rural areas, but not entering urban department, is the preferential transferring selection for the rural labor possessing the highest human capital endowment [22]. In despite that the relative poverty hypothesis could explain this problem in some degree, where the transferring decision of rural labor doesn't only depend on the expected urban-rural income difference, but also depend on the feeling relative poverty [23], the socialization evolutionary game model provides the more straight and reasonable explanation: Not only the expected urban-rural income difference has effect on the transferring decision, but also the obstacle obtaining urban employment is restricted too.

\subsection{Indirectly Knowledge Obstacle among Rural-urban Employment Departments}

The existing literatures divide Chinese rural-urban employment departments into two types of ternary structure models, where one is constituted by urban department, non-agricultural department and agricultural department in rural areas, and the other is constituted by rural department, urban formal and informal departments. The paper analyzes the quantitatively measurement problem of the knowledge transferring obstacles among Chinese rural-urban departments based on the latter ternary structure model, so gets the following knowledge transferring dynamic difference model about Chinese urban-rural ternary departments:

$$
\left\{\begin{array}{l}
\Delta \theta_{1}=\theta_{1} \cdot\left[\lambda_{2}\left(\pi_{1}-\pi_{2}\right) \cdot \theta_{2}+\lambda_{3}\left(\pi_{1}-\pi_{3}\right) \cdot \theta_{3}\right] \\
\Delta \theta_{2}=\theta_{2} \cdot\left[\lambda_{2}\left(\pi_{2}-\pi_{1}\right) \cdot \theta_{1}+\lambda_{3}\left(\pi_{2}-\pi_{3}\right) \cdot \theta_{3}\right]
\end{array}\right.
$$

Where the subscripts $1,2,3$ correspondingly denote the rural, urban informal and formal departments; and $\lambda_{1}, \lambda_{2}, \lambda_{3}$ denotes the knowledge transferring obstacles among various departments that the larger of those values, the easier of transferring into those departments.

(1) Population proportions among various ruralurban departments

We can confirm Chinese population distributing proportion $\theta_{1}, \theta_{2}, \theta_{3}$ among different rural-urban employment departments based on the statistical data within 1990-2000years (see to Appendix 1) [24], where growth rate of population isn't considered, and the population of urban formal department is approximately derived from the product of urban population and ratio between urban worker and urban employed persons.

(2) Utilities among various rural-urban departments

We can constitute the following factor game matrix based on above ternary departments model (see to Table 1 and Appendix 1), then confirm the values of factor game matrix based on the statistical data within 1990 -2000years. Now we can compute the expected utilities $\pi_{1}, \pi_{2}, \pi_{3}$ when rural labor chooses the different employment departments.

Rural dep. $s_{1} \quad$ Urban inf. $s_{2} \quad$ Urban for. $s_{3}$

\begin{tabular}{|c|c|c|c|c|}
\hline \multirow{2}{*}{\multicolumn{2}{|c|}{$\begin{array}{ll}\text { Rural dep. } & s_{1} \\
\text { Urban informal dep. } & s_{2}\end{array}$}} & $a_{1}, a_{1}$ & $b_{1}, b_{1}$ & $C_{1}, \underline{C}_{2}$ \\
\hline & & & $C_{1}$ & $\underline{\underline{e}}_{1}, \underline{\underline{e}}_{2}$ \\
\hline orma & $s$ & $\underline{C}$ & $\underline{\underline{e}}_{2}$, & \\
\hline
\end{tabular}

Table 1: The factor game of Chinese rural labor transferring.

Put above population proportions and utilities into knowledge transferring dynamic difference model about Chinese urban-rural ternary departments (7) and (8), we can indirectly measure the knowledge transferring obstacles among Chinese urban-rural various departments (see to Table 2 and Figure 1). It need pay attention that larger of values, the easier of entering this department. 


\begin{tabular}{|l|l|l|l|l|l|}
\hline Year & 1990 & 1991 & 1992 & 1993 & 1994 \\
\hline $\begin{array}{l}\text { Urban } \\
\text { informal dep. }\end{array}$ & 0.049 & 1.511 & 6.50 & 7.838 & 1.508 \\
$\begin{array}{l}\text { Urban formal } \\
\text { dep. }\end{array}$ & 0.297 & 0.267 & -0.044 & -0.311 & -0.026 \\
\hline Year & 1995 & 1996 & 1997 & 1998 & 1999 \\
\hline $\begin{array}{l}\text { Urban } \\
\text { informal dep. } \\
\text { Urban formal } \\
\text { dep. }\end{array}$ & 6.631 & 1.012 & 4.12 & 1.209 & 0.867 \\
\hline
\end{tabular}

Table 2: Knowledge transferring obstacles among Chinese urban-rural various departments in the 1990's $\left(\times 10^{-4}\right)$.

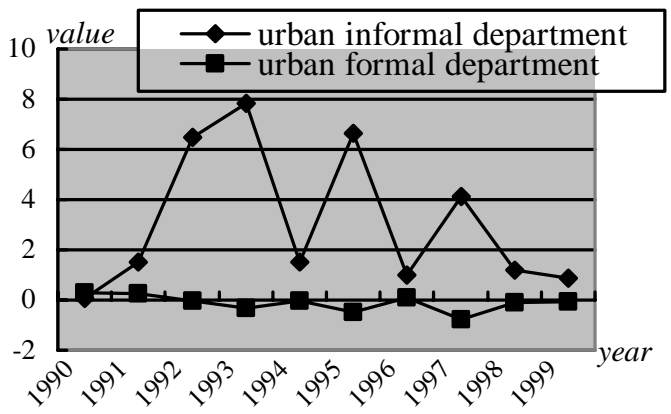

Fig. 1: Knowledge transferring obstacles among Chinese urban-rural various departments in the 1990's $\left(\times 10^{-4}\right)$.

Analysis of the computing outcome, firstly, the knowledge transferring obstacle of urban informal department is smaller than the urban formal department in the 1990's as a whole. Secondly, the knowledge transferring obstacle value of Chinese urban formal department is negative in the mostly years that shows those departments have quiet large transferring obstacle. Those departments don't only incapably absorb the surplus rural labor, but also are appear the phenomenon that the existing employed population decreases in those urban formal departments. This phenomenon can be explained by the large-scale laid-off workers in state-owned enterprises. Thirdly, after entering the metaphase of the 1990's, there has the new trend that the knowledge transferring obstacle of Chinese urban informal department is increasing too. Why? One probable explanation is some local governments carried out a series of measures to restrict rural labor entering local urban informal employment departments, in order to relieved re-employment stress of the laid-off workers in state-owned enterprises.

\section{Further Discussion}

For knowledge transferring problem, the existing literatures are mostly making the qualitative analysis or description about various complex influencing factors, and some papers are making the empirical analysis combined the corresponding survey data for the special enterprise organizational knowledge transferring problems. This paper puts forward a new quantitative method to measure knowledge transferring obstacle based on the economy exchange theory and evolutionary game theory. It can be indirectly confirmed by analysis the population distribution and corresponding utilities in some knowledge transferring course. But this analytical method still has some problems as following:

(1) This analytical idea based on the economy exchange theory strong is criticized by some sociologists.

Bouty(2000) thinks that it's worthy of suspicion that the motivation of inter-individual knowledge exchange entirely comes from the economic consideration or not [25]. The first reason, the economic worth of individual private knowledge is usually blurry in many social problems; in despite that the economic worth of rural labor transferring in this paper is clear. Secondly, single person maybe couldn't evaluate the economic benefit of knowledge exchange because of absent the corresponding information. But it is even the basic hypothesis of evolutionary game theory that is advantage neither more nor less than other fully rational analytical theories. Thirdly, the economy exchange theory ignores the social meaning in the knowledge transferring course. It's an important manner to construct the steady social relationship, or get the friendship, credit, reputation and controlling authority through exchanging the private knowledge each other. Sociologist Garnovetter puts forward the concept of embeddedness that the inter-individual exchanging behavior usually embeds into social network structure [26]. But there still is difficult to quantitative analysis for knowledge transferring obstacle, if we take the social network theory to analyze this problem. In ours evolutionary game model, the social network factors maybe indirectly hide in the leaning obstacles.

(2) The applicability of evolutionary game theory to analyze knowledge transferring obstacle

The first problem, Fudenberg points that some basic hypotheses in evolutionary game theory still restricts in the biological colony evolving category. When we apply this theory to analyze the evolutionary phenomenon in social economic complex system, there have a lot of researches need to do [27]. For example, we need construct the corresponding dynamic learning models with appropriate rational degree for some special social evolving phenomenon. As analytical foundation in this paper, general replicator dynamic is only one type of socialization 
learning model that approximately describes the social group learning course.

The second problem, the traditional evolutionary game theory supposes that the player makes the factor game where the payoff is regulated in advance. There has a problem when applied the theory to analyze practical social problems: how and who regulate the game payoff? This problem is put forward by Binmore that the practical game problem only gets one equilibrium state and others payoffs of irrational and out-of-equilibrium-path only consist in rational player's brain as the expected payoff [28]. In despite this paper takes the social economy statistical data as the corresponding payoffs among various social employment departments, this analytical method of social economy problems need make further research in the next work.

\section{Acknowledgement}

This work is partially supported by National Nature Science Foundation of China (Grant No. 70402003).

\section{References}

[1] Earl Michael and Scott Ian, What on earth is a CKO? London: London Business School Report, 1998.

[2] Nonaka and Ikujiro, A dynamic theory of organizational knowledge creation. Organization Science, 5(1):14-37, 1994.

[3] Argote L, McEvily B and R. Reagans, Managing knowledge in organization: An integrative framework and review of emerging themes. Management Science, 49(4):571-182, 2003.

[4] Xie He-feng and Shui Chang-qing, A review on informal knowledge transfer between individuals. R\&D Management, 18(4):54-61, 2006.

[5] Kim and H. Daniel, The link between individual and organizational learning. Sloan Management Review, 35(1):37-50, 1993.

[6] Hedlund Gunnar, A model of knowledge management and the $\mathrm{N}$-form corporation. Strategy Management Journal, 15:73-90, 1994.

[7] D. T. Croasdell, D. B. Paradice and J. F. Courtney, Using adaptive hypermedia to support organizational and memory. Proceedings of the 30th Annual Meeting of the Hawaii International Conference on Systems Sciences, 1998.

[8] Xu Jin-fa, Xu Qiang and Gu Jing-lei, Contextbased model of knowledge transfer in firms. Science Research Management, 24(2):54-60, 2003.
[9] Wang Zhao-xiang, A six-layer model for the knowledge transfer process. Chinese Journal of Management Science, 14(3):122-127, 2006.

[10] Peng Can, Knowledge transfer in regional innovation system: barrier and counter-move. Studies In Science of Science, 21(1):107-111, 2003.

[11] Wang Ying-luo and $\mathrm{Li} \mathrm{Xu}$, Research on knowledge transferring characteristic. Systems Engineering-theory \& Practice, 22(10):8-11, 2002.

[12] Wei Jiang and Wang Tong-an, An empirical research on the factors influencing knowledge transfer among individual, group and organization. Studies in Science of Science, 24(1):91-97, 2006.

[13] W. Weibull, Evolution, rationality and equilibrium in games. European Economic Review, 42:641-649, 1998.

[14] J. Nachbar, Evolutionary selection dynamics in games: convergence and limit properties. International Journal Game Theory, 19:59-89, 1990.

[15] R. Sethi, Strategy-specific barriers to learning and nonmonotonic selection dynamics. Games and Economic Behavior, 23:284-404, 1998.

[16] P. Haghirian, Does culture really matter? cultural influences on the knowledge transfer process within multinational corporations. Proceedings of the 11th European Conference on Information Systems, 2003.

[17] G. Szulanski, Exploring internal stickiness: Impediments to the transfer of best practice within the firm. Strategic Management Journal, 17:27-43, 1996.

[18] Uzzi B. and R. Lancaster, Relational embeddedness and learning: the case of bank loan managers and their clients. Management Science, 49(4):383-399, 2003.

[19] Eric von Hippel, Cooperation between rivals: informal know-how trading. Research Policy, 16(6):291 - 302, 1987.

[20] Perkins, Dweight H., Steven Radelet, Donald R. Snodgrass, Malcolm Gillis, and Michael Roemer, Economics of development, Fifth Edition, W. W. Norton, 2001.

[21] Hu an-gang, China needs the developing strategy of preferential employment. Decision-making Consultation, 12:4-6, 2000.

[22] Zhao Yaohui, Migration and earning difference: the case of rural china. Economic Development and Culture Change, 47(4):767-782, 1999.

[23] Cai fang and Du yang, The double motivation and political meanings of migratory: test the 
relative poverty hypothesis. Population Science of China, 4:1-7, 2002.

[24] China statistical yearbook. Beijing: Chinese statistical press, 1991 2001.

[25] I. Bouty, Interpersonal and interaction influences on informal resource exchanges between $R \& D$ and researcher across organizational boundaries. Academy of Management Journal, 43(1):50-65, 2000.

[26] M. Garnovetter, Economic action and social structure: The problem of embeddedness. American of Sociology, 91:481-510, 1985.

[27] D. Friedman, On economic application of evolutionary game theory. Journal of Evolutionary Economics, 8:15-43, 1998.

[28] K. Binmore, Foundation of game theory, advances in economic theory. Sixth World Congress, Vol.1, Edited by Laffont, J., Cambridge University Press, pp. 1-31, 1992.

\section{Appendix}

\begin{tabular}{|c|c|c|c|c|c|c|}
\hline $\begin{array}{c}\text { Propor } \\
\text { tion }\end{array}$ & $\begin{array}{c}\text { Rural } \\
\text { dep. } \\
\theta_{1}\end{array}$ & $\begin{array}{c}\text { Urban } \\
\text { informal } \\
\text { dep. } \theta_{2}\end{array}$ & $\begin{array}{c}\text { Urban } \\
\text { formal } \\
\text { dep. } \theta_{3}\end{array}$ & $\begin{array}{c}\text { Increme } \\
\text { nt of } \\
\text { rural } \\
\Delta \theta_{1}\end{array}$ & $\begin{array}{c}\text { Increment } \\
\text { of urban } \\
\text { informal } \\
\Delta \theta_{2}\end{array}$ & $\begin{array}{c}\text { Increment } \\
\text { of urban } \\
\text { formal } \\
\Delta \theta_{3}\end{array}$ \\
\hline 1990 & 0.736 & 0.013 & 0.251 & -0.005 & 0 & 0.005 \\
1991 & 0.731 & 0.013 & 0.256 & -0.006 & 0.001 & 0.005 \\
1992 & 0.725 & 0.014 & 0.261 & -0.005 & 0.006 & -0.001 \\
1993 & 0.720 & 0.020 & 0.260 & -0.005 & 0.014 & -0.009 \\
1994 & 0.715 & 0.034 & 0.251 & -0.005 & 0.006 & -0.001 \\
1995 & 0.710 & 0.040 & 0.250 & -0.015 & 0.036 & -0.021 \\
1996 & 0.695 & 0.076 & 0.229 & -0.014 & 0.010 & 0.004 \\
1997 & 0.681 & 0.086 & 0.233 & -0.014 & 0.047 & -0.033 \\
1998 & 0.667 & 0.133 & 0.200 & -0.015 & 0.020 & -0.005 \\
1999 & 0.652 & 0.153 & 0.195 & -0.014 & 0.017 & -0.003 \\
2000 & 0.638 & 0.170 & 0.192 & - & - & - \\
\hline
\end{tabular}

Data Source: Chinese statistical yearbook1991-2001

Table 1: Proportional distribution of Chinese rural labor transferring population in the 1990's

\begin{tabular}{|c|c|c|c|c|c|}
\hline $\begin{array}{r}\text { In } \\
\text {-come }\end{array}$ & \begin{tabular}{|c|} 
Annual Per \\
Capita \\
Disposable \\
Income of \\
Rural \\
Households \\
$a_{1}$ \\
\end{tabular} & $\begin{array}{c}\text { Annual Per } \\
\text { Capita } \\
\text { Disposable } \\
\text { Income of } \\
\text { Urban } \\
\text { Households } \\
b_{1} \\
\end{array}$ & $\begin{array}{c}\text { Average } \\
\text { Wage of } \\
\text { Staff and } \\
\text { Workers } \\
c_{2}, e_{2}\end{array}$ & \begin{tabular}{|c|} 
Average \\
Living \\
Expenditur \\
e of Urban \\
Poor \\
Households \\
$c_{1}$ \\
\end{tabular} & \begin{tabular}{|c|} 
Average \\
income of \\
Urban \\
informal \\
department \\
$e_{1}$
\end{tabular} \\
\hline 1990 & 686.3 & 1510.2 & 2140 & 688.9 & 720.8 \\
\hline 1991 & 708.6 & 1700.6 & 2340 & 810.7 & 811.7 \\
\hline 1992 & 784.0 & 2026.6 & 2711 & 874.0 & 967.3 \\
\hline 1993 & 921.6 & 2577.4 & 3371 & 1059.2 & 1230.2 \\
\hline 1994 & 1221.0 & 3496.2 & 4538 & 1352.2 & 1668.7 \\
\hline 1995 & 1577.7 & 4283.0 & 5500 & 1723.2 & 2044.3 \\
\hline 1996 & 1926.1 & 4838.9 & 6120 & 1936.5 & 2309.6 \\
\hline 1997 & 2090.1 & 5160.3 & 6470 & 2161.1 & 2463.0 \\
\hline 1998 & 2162.0 & 5425.1 & 7479 & 2198.8 & 2589.4 \\
\hline 1999 & 2210.3 & 5854.0 & 8346 & 2325.7 & 2794.1 \\
\hline 2000 & 2253.4 & 6280.0 & 9371 & 2325.05 & 2997.4 \\
\hline 2001 & 2366.4 & 6859.6 & 10870 & 2464.80 & 3274.1 \\
\hline
\end{tabular}

Data Source: Chinese statistical yearbook 1991 2001

Table 2: The income level of resident in Chinese urban and rural areas in the 1990’s (Unit: yuan) 